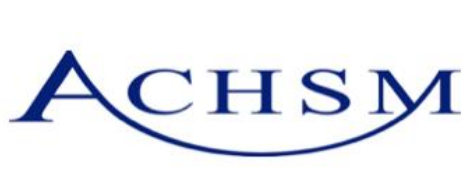

\title{
COVID-19 AND WORKING WITHIN HEALTH CARE SYSTEMS: THE FUTURE IS FLEXIBLE
}

\author{
Lea Merone, Oscar Whitehead \\ University of Queensland, Australia
}

Correspondence: lea@doctors.org.uk

\begin{abstract}
COVID-19 and subsequent lockdown of affected countries has changed the way Australia and the rest of the world do business, with online working, video/teleconferences and independent working becoming increasingly normal. Those working in primary care or in allied professions however such as administration, public health, management, human resources, radiology and mental health, have found themselves unexpectedly moving their work into their homes.
\end{abstract}

There has been much discourse surrounding the consequences and benefits of the recent work from home (WFH) massmovement. The leading benefits of working from home are increased productivity, cost and time-savings for employers and opportunities for disabled people to work. However, there are several emerging unintended adverse consequences of WFH, including overworking, stress and fatigue.

Employee personality traits are linked with the individual's response to WFH. It is the role of a good leader to play to an employee's strengths and individual circumstances. WFH initiatives can provide huge economic savings for organisations. The future beyond COVID-19 must allow for flexibility in both workers' hours and location as far as possible, with investment in telehealth and teleworking and allowance for face-to-face meetings in accommodating office-spaces.

\section{KEYWORDS}

COVID-19, health care system 
The emergence and exponential spread of novel coronavirus-19 (COVID-19) has changed the way Australia does business. [1] With World Health Organisation recommending quarantine and lockdown of affected countries, scores of workers have moved from the office to their own homes; [2] online working, video and teleconferences and independent working have rapidly become the new normal.

Healthcare systems are different from other types of organisations in that healthcare workers are often deemed to be essential. Consequentially, in terms of work location and hours, little has changed for many of these employees. Those working in primary care or in allied professions however such as administration, public health, management, human resources, radiology [3] and mental health, have found themselves unexpectedly moving their work into their homes. $[4,5]$ In the case of clinical primary care, adaptations have been rapidly made towards telehealth [5], a change which some argue is long overdue. [6]

Telehealth, particularly in rural and remote settings, comes with several benefits including: enhanced access to care, improved quality of care, lower costs and greater convenience. For clinicians, the benefits have included: access to continuing education and personal professional development (PPD), experiential learning, networking and collaboration. [7]

Whilst the benefits of telehealth are being realised, including improved cost-efficiency profiles for both organisations and patients, [8,9] there has been much discourse surrounding the consequences and benefits of the recent work from home (WFH) mass-movement and there are those who argue that WFH is probably here to stay.

Research suggests that across the USA, $37 \%$ of all jobs have the potential to be completed from home. [10] Despite this, recent survey suggests that during the COVID-19 pandemic approximately $50 \%$ of the employed US population were WFH, including $14 \%$ of these individuals who would WFH ordinarily, meaning approximately one third of workers have switched to WFH. [11] In Australia, a Gartner human resources survey demonstrated that $88 \%$ of organisations have required or encouraged employees to WFH during the COVID-19 pandemic. [12]

\section{WORKING FROM HOME: BENEFITS AND} OPPORTUNITIES

The leading benefits of working from home are increased productivity, cost and time-savings for employers and opportunities for disabled people to work [13]. Recent social media-based survey of global workers demonstrated that reception to WFH initiatives in the context of COVID-19 was resoundingly positive (73\%), with employees expressing joy, anticipation and trust. Of the $27 \%$ with a negative perception of WFH, the most commonly expressed emotions were fear, sadness, anger and distrust. [2]

Employees report feeling "trusted by their manager" when allowed to WFH, [14] leading to increased morale and wellbeing. Employee morale has been linked with greater productivity, retention and overall organisational performance. [15] WFH allows employees greater autonomy over working hours, conditions and how they manage their work and other commitments. [16] Worker autonomy is known to increase life satisfaction, particularly for female employees. [17]

Studies have also demonstrated that WFH improves worklife balance, particularly amongst millennials, who make up a large portion of the workforce [18]. A study conducted over two years reported a $13 \%$ productivity increase in those who WFH and that staff turnover decreased $50 \%$. Employees who WFH typically take shorter breaks and less sick leave. Additionally, WFH is economically preferable for employers, with reported savings of over $\$ 3000$ AUD per employee on lease costs. [19]

A recent McCrindle survey revealed that in Australia, $80 \%$ of employees would be more likely to stay longer with an organisation if provided with flexible working arrangements in terms of hours and location. This desire was strongest amongst full-time workers. Over half of employees stated they would take a pay cut for the advantage of flexible working arrangements and similarly $55 \%$ reported feeling they had increased productivity when WFH. [20]

In addition to economic costs and productivity, restricting workers to an office or workplace can be detrimental to employees' health. This is possibly attributable to commuting; long distance travel to a place of work is strenuous and strongly associated with increased incidence of obesity. [21] A study in Sweden has demonstrated that long-distance commutes are also associated with stress and marital strain, with greater 
incidence of divorce occurring in couples where at least one individual has commuted to work. [22] According to data published in the Household, Income and Labour Dynamics Australia (HILDA) survey, Australian workers spend an average of 66 minutes per day commuting. [23] WFH will obliterate the commute, allowing employees a better work-life balance and more opportunity to engage in exercise, cooking and healthier lifestyles.

Personality is a factor in work-place productivity and studies have shown that those who are extroverts have distinct advantages in the workplace. [24] Despite this, introverts make up 30-50\% of the population, [25] however they generally have lower-levels of wellbeing than extroverts and this may be because of their lack of personenvironment fit. [26] It may be that this group is better suited to a different work environment and indeed teleworking from home was pioneered by a group of people who selfidentified as introverts or 'loners'. [13]

WFH is economically optimal for organisations, research in the USA has demonstrated that each employing WFH saves their employer an average of 10,000USD in workspace and other related expenses. Additionally, remote working allows organisations to procure the talents of a wider group of workers without needing to contribute to relocation expenses. [27]

\section{WORKING FROM HOME: THE UNINTENDED CONSEQUENCES}

As boundaries between work and home life blur, research has shown that employees generally respond to WFH in one of three ways: overworking, improved planning and structure to compensate for blurring of the boundaries, or performing different tasks in the home office than the work office. [14] The blurring of boundaries and WFH can be detrimental to the mental health of some workers. A 2017 report by the European Foundation (Eurofound) for the Improvement of Living and Working Conditions determined that $41 \%$ of employees who WFH report high-levels of stress compared with $25 \%$ of employees who commute to a place of work. [28]

Organisational factors may make WFH more challenging and increase employee stress, including lack of technical and human resource support. [ 14] Loss of management culture and sense of guidance can also be impacted by WFH. [14] It is the responsibility of the good leader and manager to provide support and guidance in challenging, novel and extreme situations. [29] Additionally, lack of team cohesion whilst WFH may contribute to feelings of isolation and loneliness and compound employee stress. [30]

Research has demonstrated that WFH can decrease mental health and wellbeing for some workers. The Eurofound global study of employees who regularly or exclusively WFH demonstrated that $42 \%$ of respondents reported some level of insomnia, compared with just $29 \%$ of those who always worked in an external location. [28] This is highly likely to result in fatigue and decreased worker productivity.

Is it becoming acknowledged that WFH can be associated with fatigue and it is hypothesised that this lassitude is related to the strain of using unnatural communication devices such as videoconferencing. [31] Research suggests that whilst teleworking can improve the speed and quality of work, basic face-face contact is still needed to balance the potential adverse effects of isolation, stress [32] and fatigue.

Fatigue can also be exacerbated by stress and a sense of lack of control; this is particularly true in the context of COVID-19, where many workers are offered no other choice than to WFH, removing autonomy and sense of wellbeing for those who prefer the structured work environment.

The concerns about employee WFH regimes during lockdown are valid and concerning for both wellbeing and productivity, however there is emerging evidence that remote telehealth can play an important role in improving mental ill-health and help employees to manage distress during pandemic-related lockdown. [33]

\section{MOVING TO THE FUTURE: FUSION AND FLEXIBILITY}

Whilst there are arguments for and against strict WFH regimes during the pandemic and potentially positive implications for organisations beyond this, it is important to note there is significant individual difference between workers and the households in which they live, which will undoubtedly significantly factor into their suitability for prolonged WFH. Whilst some may find WFH improves concentration and reduces interruptions, others may have family commitments that drive them to the opposite view. 
It is the role of a good leader to play to an employee's strengths and individual circumstances. As COVID-19 develops and societal and economic structures change, the emphasis must be on flexibility in the workplace, allowing, where possible and appropriate, the freedom and trust for employees who see improved productivity and lifestyle to WFH, whilst maintaining a workplace environment for workers who for a myriad of reasons may not be suited to WFH. In the context of pandemic, this must be conducted within the context of infection control and physical distancing, which may require innovative rostering solutions to ensure the physical and mental wellbeing of employees.

Flexibility in hours has been shown to decrease absenteeism, employee turnover and work-related stress. [34] WFH initiatives can provide huge economic savings for organisations. A balance must be struck between employee wellbeing and organisational productivity. For this to happen, the future beyond COVID-19 must allow for flexibility in both workers' hours and location as far as possible, with investment in telehealth and teleworking and allowance for face-to-face meetings in accommodating office-spaces.

\section{References}

1. Mike Dockery SB. Working from home in the COVID lockdown. Bankwest Curtin Economics Centre. 2020;5.

2. Dubey AD, Tripathi S. Analysing the Sentiments towards Work-From-Home Experience during COVID19 Pandemic. Journal of Innovation Management. 2020;8(1).

3. Mahmud Mossa-Basha CCM, Danny C Kim, Michael J Tuite, K Pallav Kolli, Bien Soo Tan,. Radiology department preparedness for COVID-19: radiology scientific expert panel. Radiology. 2020;295(3).

4. Kavoor AR, Chakravarthy K, John T. Remote consultations in the era of COVID-19 pandemic: Preliminary experience in a regional Australian public acute mental health care setting. Asian J Psychiatr. 2020;51:102074.

5. Kidd M. Australia's primary care COVID-19 response. Aust J Gen Pract. 2020;49.

6. Nespolon H. New telehealth services a chance to show the real strength of general practice 2020 .
7. Jennifer J Moffatt DSE. The reported benefits of telehealth for rural Australians. Australian Health Review. 2010;34:276-81.

8. P A Jennett LAH, D Hailey, A Ohinmaa, C Anderson, R Thomas, B Young, D Lorenzetti, R E Scott, . The socioeconomic impact of telehealth: a systematic review. Journal of telemedicine and telecare. 2003;9(6).

9. Laurie Wilson. Australasian Telehealth Society - Your Health Your Say 2017.

10. Jonathan I Dingel BN. How many jobs can be done at home? National Bureau of Economic Research [Internet]. 2020.

11. Erik Brynjolfsson JH, Adam Ozimek, Daniel Rock, Garima Sharma, Hong Yi Tu Te,. Covid-19 and remote work: an early look at US data. Mimeo. 2020.

12. Vanessa Mitchell. Report- Most Australian employees to work from home CMO [Internet]. 2020 25/05/2020.

13. Joanne H Pratt. Home teleworking: a study of its pioneers. Technological Forecasting and Social Changes. 1984;25:1-14.

14. E Baker GA, J Crawford. Satisfaction and Perceived Productivity when Professionals Work from Home. Research and Practice in Human Resource Management. 2007;15(1):36-62

15. Arunchand $\mathrm{CH} H N R$. Organisational culture and employee morale: a public sector enterprise experience. Journal of Strategic Human Resource Management. 2013;2(1).

16. Yehuda Baruch NN. Home, sweet work: requirements for effective home working. Journal of general management. 1997;23(2).

17. Daniel Wheatley. Autonomy in Paid Work and Employee Subjective Well-Being Work and Occupations. 2017;44(3)

18. Heidi Lynne Kurter. 5 Ways Millennials Are Shaking Up The Workforce From The Bottom Up. Forbes [Internet]. 2018.

19. Nicholas Bloom JL, John Roberts, Zhichun Jenny Ying. Does Working from Home Work? Evidence from a Chinese Experiment. The Quarterly Journal of Economics. 2015;130:165-218.

20. MCCrindle. Teleworking in Australia- Latest Trends and Perceptions 2020 [Available from: https://mccrindle.com.au/insights/blogarchive/telewo rking-in-australia-latest-trends-and-perceptions/. 
21. Lopez-Zetina J, Lee H, Friis R. The link between obesity and the built environment. Evidence from an ecological analysis of obesity and vehicle miles of travel in California. Health Place. 2006;12(4):656-64.

22. Sandow E. Til work do us part: the social fallacy of long-distance commuting. Urban Studies. 2014;51 (3).

23. Roger Wilkins IL, Peter Butterworth. HILDA statistical report 20192019.

24. Michael P Wilmot CRW, John O Kammeyer-Mueller, Deniz S Ones,. Extraversion advantages at work: a qualitative review and synthesis of the meta-analytic evidence. Journal of Applied Psychology. 2019;104(12):1447-70.

25. Jenna Goudreau. So Begins A Quiet Revolution Of The 50 Percent2012 25/05/2020. Available from: https://www.forbes.com/sites/jennagoudreau/2012/01 /30/quiet-revolution-of-the-50-percent-introvertssusan-cain/ - 29c0767793fb.

26. Rodney Bruce Lawn. Quiet flourishing: exploring beliefs about introversion-extraversion, and identifying pathways to optimal wellbing in trait introverts: University of Melbourne; 2019.

27. OwlLabs. The State of Remote Work Report by Owl Labs USA: OwILabs; 2020 [Available from: https://www.owllabs.com/state-of-remote-work.

28. Eurofound and the International Labour Office. Working anytime, anywhere- The effects on the world of work2017.

29. Michael Holenweger MKJ, Franz Kernic,. Leadership in Extreme Situations: Springer; 2017.

30. Tammy D Allen TDG, Kristen M Shockley, . How Effective Is Telecommuting? Assessing the Status of our Scientific Findings. Psychological Science in the Public Interest. 2015;16(2).

31. Neilson K. The real reason we're all so tired: unpacking video call fatigue. HRMonline [Internet]. 2020.

32. Coenen M, Kok RAW. Workplace flexibility and new product development performance: The role of telework and flexible work schedules. European Management Journal. 2014;32(4):564-76.

33. Zhou X, Snoswell CL, Harding LE, Bambling $M$, Edirippulige S, Bai X, et al. The Role of Telehealth in Reducing the Mental Health Burden from COVID-19. Telemed J E Health. 2020;26(4):377-9.
34. Edward M Shepard TJC, Douglas Kruse,. Flexible Work Hours and Productivity: Some Evidence from the Pharmaceutical Industry A Journal of Economy and Society. 1996;35(1):123-39. 